\title{
ZU DEN ANFÄNGEN DER DEUTSCHEN SOZIALDEMOKRATIE I863-I878
}

\section{PROBLEME IHRER GESCHICHTSSCHREIBUNG}

Zahlreiche monographische Arbeiten zur sozialdemokratischen Parteigeschichte führen wohlbegründete Klage über das Fehlen einer modernen Gesamtdarstellung, die zu erarbeiten ein erstes Anliegen der Geschichtsschreibung der deutschen Arbeiterbewegung sein müsse. ${ }^{1}$ Dass es noch einer umfassenden Darstellung ermangelt, ist um so eigenartiger, als sich eine Fülle von historischen Zeugnissen und Dokumenten dem Historiker zur Bearbeitung anbietet. ${ }^{2}$ Gleichwohl erschöpft sich die bisherige parteigeschichtliche Bibliographie in Einzeldarstellungen - abgesehen von einigen gedrängten, zu parteipolitischen Zwecken geschriebenen Übersichten -, und es gilt die etwas verfrühte Rüge Adolf Mengers über die mangelhafte Geschichtsschreibung des Sozialismus heute - trotz Mehring - in erhöhtem Masse für die Geschichtsschreibung der sozialdemokratischen Partei. ${ }^{3}$

So erscheint es ebenso notwendig wie gerechtfertigt, einmal aus dem Abstand von beinahe roo Jahren jene Probleme vor allem des ersten Jahrzehnts der sozialdemokratischen Arbeiterbewegung aufzuzeigen, bei denen eine Gesamtdarstellung einsetzen könnte. Dem vorliegenden Beitrag entspricht die Frage nach dem Verhältnis von Ideologie und praktischer Politik in der sozialdemokratischen Partei. Hier stellt sich ein Problem dar, dass gerade für die Sozialdemokratie bedeutungsvoll ist. Erich Matthias hat dieser Fragestellung eine ausführliche Studie für die Zeit Karl Kautskys gewidmet, ${ }^{4}$ und Kurt Brandis bemüht sich für die vorhergehende Zeit um eine Lösung. ${ }^{5}$ Dabei leidet jedoch die Arbeit von Brandis unter ihrem stark dok-

1 In diesem Sinne siehe Erich Matthias, Zur Geschichte der deutschen Arbeiterbewegung, Neue Politische Literatur, V. (1958) S. 337 ff.

${ }^{2}$ Es sei hier nur auf die reichhaltigen Bestände des früheren Parteiarchivs der SPD, das heute zum grossen Teil im IISG Amsterdam liegt, hingewiesen.

3 Adolf Menger, Das Recht auf den vollen Arbeitsertrag, Vorrede, 1886.

4 Erich Matthias, Kautsky und der Kautskyanismus. Die Funktion der Ideologie in der deutschen Sozialdemokratie vor dem ersten Weltkriege. Marxismusstudien (I957) Zweite Folge. S. I I I ff.

5 Kurt Brandis, Die deutsche Sozialdemokratie bis zum Fall des Sozialistengesetzes (193 I). 
trinären Charakter, der sich vor allem in der Terminologie äussert (,Sozialdemokratismus") und wohl als die Folge einer nur-ideologiegeschichtlichen Betrachtung gelten kann. Um eine solche Verengung des Blickfeldes zu vermeiden, ist der vorliegende Versuch darauf getichtet, die Ideologie nicht an ihr selbst, sondern an ihrer historischen Umgebung zu messen, um von hier aus ihre Möglichkeiten zu erkennen.

In der Tat wuchs doch die Sozialdemokratie in einer Welt auf, in der sich gerade die Mazzinische Revolution vollzog, die russische junge Intelligenz „nach vorn” strebte, Zar Alexander II die Leibeigenschaft aufheben musste und der amerikanische Sezessionskrieg in sozialistischen Kreisen eine klassenkämpferische Deutung erfuhr. In Preussen bestimmte nach dem Versagen des liberalen Bürgertums in den Jahren I 848/49 die oktroyierte Verfassung das konstitutionelle Leben. Hier wie auch in anderen Staaten Deutschlands wich die fruchtbare politische Dynamik, wie sie in den Revolutionsjahren in Ansätzen zu erkennen gewesen war, einem im Hinblick auf die Ziele der letzten Jahre politisch sterilen Leben. Dabei verlagerte sich die staatspolitische Bemühung des liberalen Bürgertums auf den wirtschaftlichen Bereich. Man überwand das politische Anliegen der Vorjahre, um teilzuhaben an einer wirtschaftlichen Prosperität, die die Depression der 40-er Jahre ablöste und sich nun im zweiten sogenannten Kondratjew-Zyclus kundtat, und es ist interessant zu erfahren, dass Rochau gerade in diesen Jahren den Begriff der „Realpolitik” prägte.

Die erste Phase der Weltkonjunktur äusserte sich in einem völligen Umbau des Verkehrswesens. Man denke nur an den fieberhaften Bau von Eisenbahnnetzen und die Verdrängung der Segelschiffe durch Dampfboote. Zwar lagen die eigentlichen Ursachen der günstigen Wendung in Deutschland ausserhalb der nationalen Sphäre, jedoch wird man beachten müssen, dass die Beseitigung letzter feudaler Überbleibsel die Volkswirtschaft empfindlicher reagieren liess und es nicht zuletzt der Zollverein war, der die Dinge in eine glückliche Entwicklung trieb. Produktion und Handel mehrten sich in raschem Tempo. Die Steinkohlenförderung stieg zwischen 1848 und 1864 ungefähr um das 5 -fache und die Eisenproduktion im gleichen Zeitraum um das 4-fache. Zählte die Firma Krupp 1851 noch 704 Arbeiter, so waren I 86I bereits 2.000 und 1873 sogar 6.000 Menschen hier beschäftigt. Einen ähnlichen Zuwachs an Arbeitskräften verzeichnete die Lokomotivenfabrik Borsig. ${ }^{1}$

${ }^{1}$ Die statistischen Angaben sind bei A. Sartorius von Waltershausen, Deutsche Wirtschaftsgeschichte 1815-1914 (1923) $)^{2}$ nachzuschlagen. 
Der günstigen Entwicklung der Schwerindustrie stand die ungleich schwierigere Lage der Leichtindustrie gegenüber. Von einer expansiven Gestaltung dieses Wirtschaftszweiges konnte hier kaum die Rede sein, vielmehr vollzog sich hier ein wirtschaftlicher und sozialer Prozess, in denen Handwerk und Heimindustrie zum grossen Teil untergingen. Das bedeutete zugleich eine fortschreitende Proletarisierung der Handwerkerschaft, die nunmehr im Fabrikproletariat aufging.

Dieser in der ökonomischen Entwicklung liegende Untergang der kleinen Einzelbetriebe zeigte sich in den folgenden Jahren nicht nur sozial bedeutsam, sondern wog auch politisch schwer in den Jahren der neuen Parteigründungen nach I 860. Der Handwerker, vor allem der Geselle, wesentlicher sozialer Typus der 48-er Jahre, starb aus. Politisch hiess das, dass auch die radikale Demokratie aus den vergangenen Revolutionsjahren immer mehr an Boden verlor und schliesslich als mögliche Parteiformation ausfiel. Zur selben Zeit zog es das liberale Bürgertum weiter nach rechts, die Arbeiterschaft schliesslich in linke sozialistische Organisationen.

Die Überwindung der ständig wachsenden Kluft zwischen den beiden Fronten wäre einer kleinbürgerlich-demokratischen Partei zugefallen. In der Volkspartei war der Ansatz hierzu gegeben - wenn man einmal von ihrer Animosität gegen Preussen absieht und sie nur auf den sozialen Gehalt ihres Programms hin untersucht. Aber die rasche Entwicklung und die entschiedene Haltung der anderen Parteien schaltete gleich jede Kompromissmöglichkeit aus. Und nichts hat schliesslich das Versagen der Volkspartei deutlicher machen können als der Ubertritt Johann Jacobys zu den Eisenachern. ${ }^{1}$

Dieser Prozess, den Gustav Mayer in einem instruktiven Aufsatz als die "Trennung der bürgerlichen von der proletarischen Demokratie" bezeichnete, ${ }^{2}$ bedeutete eine Auflösung der gemeinsamen demokratischen Volksbewegung aus den Jahren der 48-er Revolution, hinter der alle Klassen standen. Der politische Gedanke stand für die 48-er Revolutionäre im Vordergrund, und hinter ihm blieben zunächst alle sozialen Widersprüche verborgen. Sie traten nun zutage in dem Masse, wie sich die politische Front in Wirtschaftsfronten auflöste. Dass die Begriffe "Liberalismus" und „Demokratie" im Zuge dieser Entwicklung ein neues Verständnis erfuhren, stellte sich als notwendige Folge dar. Zwar waren auch schon 1848 beide

${ }^{1}$ Inwieweit die allgemeine politische Einstellung die Volkspartei scheitern liess, soll hier ausser Acht gelassen werden,

${ }^{2}$ Vgl. Gustav Mayer, Die Trennung der proletarischen von der bürgerlichen Demokratie in Deutschland (1863-1870). In: Archiv für die Geschichte des Sozialismus und der Arbeiterbewegung, II S. I ff. 
Begriffe verschiedenen Inhalts gewesen - das fand seinen politischen Ausdruck in den Forderungen der demokratischen Linken gegenüber den liberalen Märzministerien - aber man wird nicht verkennen dürfen, dass damals „liberales” oder ,demokratisches” Handeln vornehmlich Kampf um gemeinsame Ziele, die politischen Freiheiten nämlich, bedeutete. Das änderte sich in den 6o-er Jahren völlig. Nunmehr wurde die politische Frage mit dem sozialen Problem des vierten Standes belastet, und die Ausdeutung des Wortes „liberal" war so restriktiv, dass selbst das allgemeine Wahlrecht keinen Platz mehr fand, während umgekehrt „demokratisch" jetzt nicht nur politisch, sondern auch "sozial-demokratisch" verstanden wurde.

Unter solchen historischen Voraussetzungen - im Zuge einer allgemeinen Parteienbildung - vollzog sich der Eintritt sozialistisch gesinnter Gruppen in das politische Leben. So wird auch eine Geschichtsschreibung jener sozialistischen Parteien - und hier sei etwas zur Periodisierung gesagt - mit den Jahren der Parteibildung einsetzen müssen. Das würde eine Revision des Mehringschen Entwicklungsschemas bedeuten, der mehr als die Hälfte seiner umfangreichen Parteigeschichte den Vorläufern widmet. Mehrings Periodisierung erweckt den Eindruck, als ob zwischen frühsozialistischen Ideen und sozialdemokratischer Parteibildung ein kontinuierlicher Faden bestehe. Es ist dabei interessant zu wissen, dass Karl Marx, wohl merkend, dass man in der Parteibewegung nicht das richtige Verhältnis zu den sozialistischen Bünden der Vergangenheit finden konnte, noch I876 im „Volksstaat" schrieb, man müsse die deutsche Arbeiterschaft immer wieder auf den „Bund der Gerechten” und den „Kommunistenbund” als die traditionsreiche Grundlage der jetzigen Parteibewegung hinweisen. ${ }^{1}$ Diese Verbindung wird man jedoch nicht anerkennen können. Vielmehr scheint uns, dass vornehmlich ein Studium der liberalen Entwicklung in ihren wirtschaftlichen, sozialen und ideologischen Folgen die Anfänge der sozialdemokratischen Parteibewegung der 6o-er Jahre deutlicher werden lässt. Wie wenig man sich in den Reihen der Sozialdemokratie einer Kontinuität der Bewegung bewusst war, das drückt doch August Bebel in seinen Lebenserinnerungen aus, ${ }^{2}$ und wie wenig fundiert sich noch das Selbstverständnis der Arbeiterschaft als Klasse gegenüber dem Bürgertum zeigte, wusste Julius Vahlteich rückschauend in die treffenden Worte zu kleiden: „Wie schon gesagt, waren unsere Kenntnisse auf dem Gebiete der Volkswirtschaft gering. Wir wussten nichts vom konstanten und variablen Kapital und waren nicht eingeweiht in die tiefsten Geheim-

1 „Der Volksstaat”, 27.2.1876.

2 August Bebel, Aus meinem Leben, I (1910) S. so. 
nisse der Kapitalbildung. Aber dass das Kapital und die Kapitalisten unsere Feinde waren, wussten wir ganz genau, und wir wussten auch, dass wir sie nur durch Machtentfaltung, also durch Organisation überwinden konnten. Wir wussten nichts vom relativen Mehrwert und hundert anderen ökonomischen Kategorien... aber wir waren ganz sicher in der Überzeugung, dass wir von den Unternehmern betrogen und bestohlen würden und dass die Gesetze dazu gemacht waren, diese Handlungsweise zu sanktionieren." ${ }^{1}$ Der Sozialismus wurde hier eher gefühlsmässig empfunden als wissenschaftlich erkannt.

\section{II}

Die sich im Zuge der industriellen Revolution vollziehende, wesentlich wirtschaftliche Orientierung der Gesellschaft und die damit verbundenen nationalpolitischen Bestrebungen in Preussen-Deutschland fanden schliesslich ihren Niederschlag in Parteigruppicrungen, deren programmatische Erklärungen zusammengenommen durchaus geeignet sind, ein Gesamtbild der politischen und sozialen Bedürfnisse in Deutschland zu vermitteln.

Ungleich der englischen Entwicklung nun, in der die politische Partei als selbstverständliches innenpolitisches Element auftrat, vollzog sich der Eintritt der deutschen Parteien in das konstitutionelle Leben nicht als notwendig anerkannter Vorgang parlamentarischen Lebens, ${ }^{2}$ sondern geschah unter Voraussetzungen, die von Tradition und „realpolitischen" Bedürfnissen gleichermassen belastet waren. Karl Marx, der englisches Parteileben aus nächster Anschauung miterleben und auf eine traditionsreiche Geheimbündelei zurückblicken konnte, schreibt einmal in einem Brief an Ferdinand Freiligrath, dass er unter Partei immer die Partei im grossen historischen Sinne verstanden habe $!^{3}$ Was unter „Partei im grossen historischen Sinne” zu begreifen war, wird man aus einer anderen Stelle desselben Briefes entnehmen dürfen. Dort heisst es: „Der 'Bund', wie die société des saisons zu Paris, wie hundert andere Gesinnungsgruppen, war nur eine Episode in der Geschichte der Partei, die aus dem Boden der Gesellschaft naturwüchsig sich bildet". ${ }^{4}$ Dass man überhaupt von

1 Das Leipziger Zentralkomitee und Ferdinand Lassalle. In: Die Gründung und Entwicklung der deutschen Sozialdemokratie. Festschrift d. Leipz. Arbeiter zum 23. Mai 1913. Hrsg. v. Bezirksvorstand der sozialdemokratischen Partei Leipzigs. S. I6.

2 Vgl. Carl Jantke, Der vierte Stand. Die gestaltenden Kräfte der deutschen Arbeiterbewegung im 19. Jahrhundert, (1955) S. 127.

${ }^{3}$ Franz Mehring, Freiligrath und Marx in ihrem Briefwechsel. Ergänzungshefte zur Neuen Zeit, ${ }^{12}$. S. 46. 29.2.1860. (Ig12).

Ebd. S. 43 . 
Partei als organisierter Gesinnungsgemeinschaft redete ${ }^{1}$ - die Organisation des Kommunistenbundes und die der "Partei im grossen historischen Sinne" wird man dabei lediglich graduell oder quantitativ verschieden sehen dürfen - machte eben einen Unterschied der westeuropäischen Länder zu dem mitteleuropäischen Deutschland. Und sicherlich übte bei Marx das englische Beispiel und eine gewisse Tradition der Geheimbündelei starken Einfluss aus.

In Deutschland lief neben der innerparteilichen Diskussion um die Verwirklichung der politischen Wünsche zugleich viel gewichtiger die Auseinandersetzung um das parteipolitische Prinzip in seinem Verhältnis zum bestehenden Staat. Solche Fragen zu beantworten, machten sich die Liberalen vornehmlich zur Aufgabe. Ihr Urteil über die eigene Stellung wie über die der sozialistischen Parteien orientierte sich an der traditionsträchtigen Geschichte des Liberalismus und gab der Frage nach dem politischen Bedürfnis nur wenig Raum. So sammelten sich die Parteien gleich zu Beginn in starker ideologischer Distanz. ${ }^{2}$ Die insbesondere von Treitschke in den Preussischen Jahrbüchern geführte Auseinandersetzung legt hierfür beredtes Zeugnis ab. Es ist ein bemerkenswertes Zeichen, dass die Sozialdemokratie im Zuge jener Diskussion gleichsam am Rande abgetan wurde. Und wenn Parisius Polen und Sozialdemokraten in einem Atemzug als nationalfeindlich bezeichnet, so ist damit über die schwierige Ausgangssituation der sozialdemokratischen Bewegung schon manches ausgesagt. ${ }^{3}$ Die Liberalen und natürlich auch die Konservativen sahen sich im Zentrum des Staatslebens. Die Sozialdemokraten nach aussen abdrängend, wurde der liberale Staatsbegriff an den konservativen Gegebenheiten gemessen, und man trachtete zugleich, die soziale Frage ohne Mitwirken der Sozialdemokraten zu lösen. Dabei darf hier nicht unerwähnt bleiben, dass in den ersten Jahren die soziologische Struktur der Parteien die Liberalen durchaus in ihrem Anspruch auf Lösung der sozialen Frage bestärken konnte. Die Grenzen zwischen Kleinbürgertum und Arbeiterschaft waren noch fliessend, und das Bewusstsein der Arbeiterschaft zeigte sich noch nicht als wissenschaftlich unterbautes Selbstverständnis einer Klasse. Und hier lag ja der Punkt, an dem

1 Es liessen sich dazu noch zahlreiche Beispiele vor allem der früheren Jahre anführen. ${ }^{2}$ So auch richtig Carl Jantke, a.a.O. S. 127. Jantke weist auch auf den englisch-deutschen Unterschied hin, wenn er schreibt: „Die deutschen Parteien waren weithın auf Prinzipien, die englischen auf politische Bedürfnisse gegründet."

3 Dass zur Zeit des Sozialistengesetzes die Sozialisten "mit sonstigem üblem Gelichter" gleichgesetzt wurden, ist wohl nicht allein der politischen Erregung zuzuschreiben. Die Wurzeln solcher Synonyme liegen viel tiefer! 
liberale Reformer wie Schulze-Delitzsch ansetzen konnten. ${ }^{1}$ Es soll nicht verkannt werden, dass auch praktisch-politische Gesichtspunkte die Haltung der Liberalen gegenüber den Sozialisten bestimmten - wirtschaftlich utilitaristisches Denken drängte sich immer mehr in den Vordergrund - aber man wird darüber hinaus nicht jenen Einfluss altliberaler Tradition übersehen dürfen, der die Liberalen in ihren eigenen Reihen zu einer Auseinandersetzung mit den frischen positivistischen Tendenzen zwang. Treitschkes und Baumgartens Erörterungen müssen vor allem von hierher verstanden werden. Altliberales Gedankengut über Mass und Möglichkeit einer politischen Partei entwickelte sich in der Zeit des Vormärz, in der schon die Staatslehre einer Theorie der Partei entgegenwirkte. ${ }^{2}$ Der Staat wurde im Hegelschen Sinne als sittliches Ganzes begriffen, und blosses „Meinen und Räsonnieren” fanden als Ausdruck subjektiven Interesses keinen Platz. Es ist unzweifelhaft, dass dieser Gedanke vom Staat als sittlichem Ganzen - wenn auch konservativen Ursprungs - in der Auseinandersetzung der Liberalen mit den Sozialdemokraten wiederaufgenommen wurde. Zugleich aber knüpfte man an Versuche einer Partei-Definition an, wie sie bei Ruge begannen und hin zu Haym und Treitschke reichen. Verstand Ruge noch die Partei als eine notwendige Antithesis zum bestehenden Staate, schloss er damit noch „Parteien-Pluralismus” aus, so ist bei Haym schon das „Prinzip der Meinung” festzustellen, und Treitschke schliesslich geht einen Schritt weiter, indem er hinter der ,subjektiven Meinung” ein „objektives Interesse” entdeckt, wenn er sagt: „Die politische Theorie greift heute in die Wandlungen des Parteilebens tiefer ein als vormals in naiveren Zeiten; aber sie kann selten parteibildend wirken, wenn sie nicht den Interessen einer sozialen Macht entspricht. Namentlich die Interessen der Gesellschaftsklassen sind mit den Parteilehren weit fester verflochten, als die Parteien selber zugeben." 3 Treitschke ist Realpolitiker genug, um nicht die Kompliziertheit der Verhältnisse in der aufkommenden Industriegesellschaft zu übersehen, aber der Anerkennung der verschiedenen Interessengruppen (Parteien) als Glieder eines dynamischen Staatsganzen setzt er Grenzen. Staatsgesinnung als tätige Kraft und Wahrung der

1 Vgl. Ernst Schraepler, Linksliberalismus und Arbeiterschaft in der preussischen Konfliktszeit. In: Forschungen zu Staat und Verfassung. Festgabe für Fritz Hartung, (1958) S. 387 .

2 Hierzu und für die folgenden Ausführungen siehe vor allem Theodor Schieder, Die Theorie der Partei im älteren deutschen Liberalismus. In: Aus Geschichte und Politik. Festschrift für Ludwig Bergstraesser, (1954) S. I $84 \mathrm{ff}$.

${ }^{3}$ Der ausgesprochene Klassencharakter der liberalen Parteien wird erst im ausgehenden I 9. Jahrhundert zugegeben. Zur Haltung Naumanns in dieser Frage vgl. Schieder, a.a.O. S. 195 . 
Macht des Staates durch die Tat des Volkes selbst sind ihm wesentliche Komponenten, die sich am Ordnungsgedanken orientieren. ${ }^{1}$ Wie fühlbar ist bei ihm doch hier Rankescher Einfluss, wie sehr setzt sich doch das Prinzip des ,juste milieu” durch. Bei aller Anerkennung der Notwendigkeit politischer Parteien wird bei ihm doch politisches Leben weit mehr vom Staate als von der Gesellschaft her begriffen, und auf diesem Hintergrunde muss das Wissen um den Machtdrang der Parteien die Skepsis des politischen Analytikers noch erhöhen. ${ }^{2}$

Wie wenig man tatsächlich einzelne Gesellschaftsklassen und Staat als miteinander verflochten sehen will, lässt ein Aufruf der Fortschrittspartei deutlich werden, in dem es unter Artikel IV heisst: „Abweisung jeder Ausbeutung des Staates für die Sonderinteressen einzelner Gesellschaftsklassen, mögen dieselben seitens der bisher privilegierten Stände oder seitens der sozialistischen Arbeiterparteien geltend gemacht werden." 3 Sieh hier die entschiedene Ablehnung des Klassenstaatsgedankens, wobei jedoch nicht übersehen werden darf, dass man sich durchaus der eigenen „Klassensituation” bewusst ist. Erst drei Jahrzehnte später ermunterte Friedrich Naumann die Liberalen, das doch zu bekennen, was zu sein ihnen der Demokrat Julius Fröbel schon 186I bescheinigte, wenn er schrieb: „Ubrigens ist der Liberalismus, wie er im europäischen Volks- und Staatsleben gegenwärtig die grosse Rolle spielt, keineswegs als das System der Volksfreiheit im Allgemeinen zu verstehen, sondern ist ein System im besonderen Interesse ganz bestimmter Elemente der Gesellschaft, welche sich im handeltreibenden und industriellen Mittelstande zusammendrängen. Der liberale Staat, in diesem conventionellen Sinne, ist der Staat, welcher dem Vorteile dieser Menschengruppe entspricht, womit keineswegs gegeben ist, dass er auch dem Vortheile aller anderen Volksclassen oder auch nur dem Vortheile der wahren Mehrheit des Volkes entsprechen müsse." 4 Wie weit man sich in liberalen Kreisen auch schon zu Fröbels Zeiten dieser Tatsache bewusst war, beweist das Verhalten von Unruhs und Rochaus gegenüber den in Arbeitervereinen zusammengeführten unteren Klassen, denen schliesslich jeder politische und sittliche Wert abgesprochen

1 Vgl. H. von Treitschke, Historisch-politische Aufsätze III (1871) S. 436 u. 443. 3. Auf.

2 Ebd. S. 445 heisst es: „Die bewegende Kraft der Parteiung ist heute noch wie vor Jahrtausenden nicht das Bekenntnis, sondern der Drang nach Herrschaft. Nicht das idem sentire de re publica schaart die Parteien zusammen, sondern das idem velle..." ${ }^{3}$ Ludolf Parisius, Deutschlands politische Parteien und das Ministerium Bismarck (1878) S. I32.

4 Julius Fröbel, Theorie der Politik, als Ergebnis einer erneuerten Prüfung demokratischer Lehrmeinungen, I (I861) S. 258. 
wurde. ${ }^{1}$ Hier wird man noch einmal auf den eingangs erwähnten Zusammenhang zwischen idealistischem Prinzip und utilitaristischem Denken verweisen müssen. Die zunehmende Politisierung der sozialen Frage nämlich, die von verschiedenen Seiten vorgebrachten Lösungsmöglichkeiten des sozialen Problems drängten die Liberalen immer mehr auf eine Klassenposition zurück. Die Organisierung der Arbeiter in sozialistischen Parteien (Lassalle, Eisenacher) war ein nur zu deutlicher Hinweis darauf, dass das manchesterliche „laissez faire" nicht mehr genügte. Dabei bemühten sich nicht nur die sozialistischen Parteien, sondern auch Volkswirtschaftler des Kreises um Schmoller und Lujo Brentano, die des gesellschaftlichen Umsturzes wahrlich unverdächtig waren, um eine gerechte Lösung. Dass man in diesen Kreisen ein Sozial- und Wirtschaftsprogramm aufstellte, welches mit den manchesterlichen Prinzipien keineswegs übereinkam, war für die Liberalen (Bamberger) Anlass genug, auszusprechen: „Ein gemeinsames Band umschlingt alle Schattierungen von dem wilden Russen Bakunin bis zum zahmsten deutschen Dozenten."2 Dabei beliess es die liberale Kritik nicht bei einer grundsätzlichen theoretischen Ablehnung. Bamberger vielmehr versuchte, die sozialistische Idee an ihren grundsätzlichen politischen Möglichkeiten zu messen. Dem sozialistischen Programm stellte er einfache tagespolitische Forderungen gegenüber, daran gemessen erschien ihm das Programm als ,soziale Alchemie". So konnte er schreiben: „Daher bleibt alle Gesellschaftsrettung nach plötzlich erfundenen Rezepten, soweit sie nicht geradezu Betrug ist, wilde Fantasterei. Daher auch vermöchte derjenige Sozialismus, der mehr als eine humanistische Tendenz sein und sich zu einem ganz und gar die Gesellschaft umformenden System erheben will, erst dann ernstlich mitzusprechen, wenn er sich einmal in praktische Forderungen eingelebt hätte, die ihrerseits in ganz elementaren Anschauungen des allgemeinen Bewusstseins sich durchgearbeitet haben müssten". ${ }^{3}$ Bamberger stellte die Frage nach den konkreten Möglichkeiten des Sozialismus und belastete dabei die Geschichte des Sozialismus und somit die Sozialdemokratie nicht nur mit der vorrevolutionären Zeit der Russen Bakunin und Tschernyschewsky, sondern auch mit den fehlgeschlagenen Pariser Versuchen. ${ }^{4}$ Das bedeutete nicht allein eine Verweisung der Frage in den internationalen Bereich, sondern auch eine Überschätzung „der ideologischen und revolutionären Elemente

1 Dazu mehr unten Abschnitt III.

2 Angefüht bei Walter Gagel, Die Wahlrechtsfrage in der Geschichte der deutschen liberalen Parteien 1848-1918. (1958) S. 80.

${ }^{3}$ Ludwig Bamberger, Politische Schriften von I 848 bis 1868 . (1895) S. 242.

4 Ludwig Bamberger, Politische Schriften von 1868 bis 1878 . (1896) S. 48. 
im Programm der Sozialdemokraten."1 Das war bedeutsam für die Haltung der Liberalen. Dem Gedanken des internationalen gesellschaftlichen Umsturzes wurde der Wille, den nationalen Staat zu erhalten, entgegengesetzt, wobei sich wohl mit Fug die Ansicht vertreten lässt, dass diese Alternative kaum den realen politischen Möglichkeiten entsprach und im letzten nur eine Verengung des Blickfeldes auf Seiten der Liberalen bedeutete. Dass die staatserhaltende Aufgabe nur dem Bürgertum zugewiesen werden konnte, galt als selbstverständlich. Hans Baumgarten, liberaler Zeitgenosse Treitschkes und Bambergers, vertritt in den Preussischen Jahrbüchern diese Auffassung, wenn er darauf hinweist, dass „das Bürgertum seiner Intelligenz, seines Fleisses, seines Reichthums und seines fast einmüthigen Willens sich stolz bewusst", endlich die Stellung im öffentlichen Leben erringen wollte, „die ihm, wie es gar nicht zweifelte, gebühre."2 Es wird deutlich, dass hier das Bürgertum in einer durch Besitz und Bildung bestimmten Klassenposition gesehen wird. Bürgerliches Erwerbs- und Erfolgsstreben füllen den gesellschaftlichen Raum, in dem die Bildung als zwischen den Klassen vermittelndes Band ihren Platz finden sollte. Die Geschichte der Arbeitervereine zeugt davon. ${ }^{3}$

Einer solchen Auffassung der eigenen sozialen Situation war eine aristokratische Tendenz immanent, und es nimmt darum nicht wunder, wenn Baumgarten der ,geschichtlichen Tat des Einzelnen" einen besonderen Platz zuordnet, wenn für ihn die Demokratie, des Hauptes" bedarf, ${ }^{4}$ und so konnten hochentwickeltes bürgerliches Selbstbewusstsein sowie ganz reales Klasseninteresse dem allgemeinen Wahlrecht auch nicht zustimmen. ${ }^{5}$

Gegenüber einem solchen Persönlichkeitsbegriff, wesentlich bestimmt durch Leistung und Bildung, nahm sich für Treitschke die sozialistische Zielsetzung als "matter Eudämonismus” oder ,nackte Sinnlichkeit" aus, und es ist spürbar, wie sich in einigen liberalen Kreisen die soziale Frage lediglich als Magenfrage stellte. Es ist aber zugleich nicht zu verkennen, dass die Ablehnung des Sozialismus im Sinne der kantischen Ethik ${ }^{6}$ um so schwieriger wurde, je mehr sich Männer wie Johann Jacoby und Friedrich Albert Lange über den „kategorischen Imperativ" der Arbeiterbewegung zuwandten.

1 In diesem Sinne argumentiert auch Walter Gagel, a.a.O. S. 86.

2 Hans Baumgarten, Der deutsche Liberalismus, Preussische Jahrbücher, 18, II, S. 592.

3 Darüber unten Abschnitt III.

4 Baumgarten, a.a.O. S. 600.

5 Zur Haltung der Liberalen zum allgemeinen Wahlrecht s. Gagel, a.a.O. S. $90 \mathrm{ff}$.

6 Vgl. ebd. S. 85 . 


\section{III}

Die neuen revolutionären Forderungen, die mit dem Kommunistischen Manifest zum Kontinent herüberkamen, trafen in Deutschland auf wenig fruchtbaren Boden. Wenn Marx geglaubt hatte, trotz der eingestandenen Schwäche der sozialen Opposition in Deutschland eine Revolution gegen den „Feudalismus” als eine gemeinsame Sache von Bürgertum und Proletariat erwarten zu dürfen, so musste er sich getäuscht sehen. Der Aufstand des Bürgertums schlug fehl - die mögliche Rolle des Proletariats war in den 48er Jahren kaum zur Sprache gekommen. Die Demonstration der Gesellen in Frankfurt blieb politisch ungesehen. Die folgenden Jahre bedeuteten einen politischen Rückschlag. Das Bürgertum, das versagt hatte, fand seinen Ausweg im wirtschaftlichen Bereich. Von der Bildung einer politischen Partei konnte zunächst keine Rede sein, und i 854 erliess der Bundestag ein Vereinsgesetz, durch dessen $\ 8$ sämtliche Bundesregierungen verpflichtet wurden, ,die in ihren Gebieten etwa noch bestehenden Arbeitervereine und Verbrüderungen, welche politische, sozialistische und kommunistische Zwecke vetfolgen, binnen zwei Monaten aufzuheben und die Neubildung solcher Vereine bei Strafe zu verbieten."1

Auf solcher gesetzlicher Grundlage stellte sich dann am Ende der soer Jahre das Arbeiterproblem aufs neue. Führende Persönlichkeiten des Nationalvereins taten die ersten Schritte, um auf dem Wege über die Arbeiterbildungsvereine die Arbeiterschaft in die liberale Bewegung einzubeziehen. Es lag nicht in der Absicht der Liberalen, auf diese Art eine selbständige politische Arbeiterbewegung zu stützen, wie es das Kommunistische Manifest noch gehofft hatte - die Grundtendenz der Vereine war vielmehr, die Arbeiterschaft von der politischen Tätigkeit hinwegzuführen. Das entsprach durchaus dem politischen Ziel, das Hermann Schulze-Delitzsch in seiner Schrift „Arbeiterfrage” gleichsam als Leitsatz aufstellte, wenn er formulierte: „Zeigen wir in der Arbeiterfrage das wahrhaft Konservative unserer Grundsätze, leiten wir immer mehr den Strom der Arbeiterbewegung von jenen verderblichen sozialpolitischen Träumereien ab auf wahrhaft erprobte volkswirtschaftliche Bahnen...", und im Jahresbericht des Erfurter Vereins von I 862 hiess es, man habe den Verein „zu dem Zwecke gestiftet, allgemeine Bildung unter den gewerbetreibenden und arbeitenden Klassen zu verbreiten."3

1 BVG. 13.7.1854. Sonderdruck 1865 .

2 Hermann Schulze-Delitzsch, Schriften und Reden. Hrsg. von F. Thorwart. II (I gro) S. I s. ${ }^{3}$ In „Koburger Arbeiterzeitung”, 25.1.1863. Angeführt bei Karl-Heinz Leidigkeit, Wilhelm Liebknecht und August Bebel in der deutschen Arbeiterbewegung 1862-1869 (1957) S. II. 
Es wäre dabei verfehlt, wollte man die für die Mitglieder in ihrer Tätigkeit unpolitischen Vereine allein unter dem Aspekt ,realpolitischer Nützlichkeitserwägungen” liberaler Interessengruppen betrachten. Zu sagen, die Vereine sollten lediglich eine Schanze in der Abwehr „sozialpolitischer Träumereien" sein, berührt zwar ein wesentliches Moment, löst aber kaum das ganze Problem. Sicherlich musste das liberale Bürgertum ein machtpolitisches Interesse daran haben, die Arbeiterschaft in seiner Gedankenwelt festzuhalten und den heftigen Angriffen Lassalles gegen das manchesterliche System zu begegnen. Jedoch wird man das erst für ein späteres Stadium geltend machen können, zu einer Zeit, in der die polemische Auseinandersetzung eine Persönlichkeit wie von Unruh zu der pauschalen Verurteilung der Arbeiterschaft hinsichtlich ihrer kulturellen und politischen Fähigkeiten zu verleiten vermochte. Erst die Opposition gegen Lassalle zwang die Fortschrittler in diesen rein politischen Weg. ${ }^{1}$

So mag ein erster Blick Persönlichkeiten wie Friedrich Albert Lange und Franz Ziegler gelten, die sich durchaus als eifrige Anhänger der „bürgerlichen Arbeiterbewegung” zeigten. Der Kantianer und der Altliberale werden sicherlich als der nüchternen politischen Spekulation unverdächtig erkannt werden. Neben ihnen wurde noch zahlreiche andere warmherzige Vertreter des Bürgertums angezogen, die hier ein dankbares Betätigungsfeld fanden. Eine Stelle aus einem Brief Zieglers nun an Lassalle vermag recht eigentlich die Grundhaltung wiederzugeben, die solche Kreise an die Arbeiterbildungsvereine herangeführt hat. So schrieb Ziegler im Februar 1863: „Ich war Student, als ich nach Garves Ubersetzung den ersten Satz von Smith las: ,Die Arbeit, welche jede Nation jährlich verrichtet, ist der Fond, der sie ursprünglich mit allen von ihr jährlich verbrauchten Notwendigkeiten und Bequemlichkeiten des Lebens versorgt."' 2 Für Ziegler bedeutete dieser Satz des liberalen Wirtschaftstheoretikers jedoch nicht Identifizierung der Arbeit mit dem Arbeiterstand allein; ihm, dem Individualisten, gab er vielmehr die Möglichkeit zur völligen Objektivität gegenüber der Gesellschaft, die ihm weniger klassenkämpferisch geschieden erschien, sondern sich vielmehr als eine Gemeinschaft „Selbstverantwortung tragender Individuen” darstellte. Das war nicht jene übermässige Begeisterung, die dem Arbeiterstand nunmehr innerhalb der Gesellschaft eine privilegierte Stellung zuerkannte, sondern - und hierzu liesse sich noch eine Viel-

` Vgl. dazu Hildegard Reisig, Die Rolle der Bildung für die Befreiung des Proletariats, phil. Diss. (1933) S. 88.

${ }^{2}$ Ferdinand Lassalle, Nachgelassene Briefe und Schriften, V (1925) S. 92 ff. 27.2.1863. 
zahl von Zitaten anderer Persönlichkeiten der bürgerlichen Seite anführen - eine nüchterne Betrachtungsweise, ${ }^{1}$ die jedem in der liberalen Gesellschaft gleiche Startchancen geben wollte und im Grunde auch schon gegeben sah. Auf diesem Boden wuchs der Gedanke der Selbsthilfe - bei Schulze-Delitzsch zunächst noch vornehmlich für die Handwerkerschaft gedacht - zum Motto der Arbeiterbildungsvereine heran. Dieser Gedanke implizierte ein Mehrfaches: die ganz konkrete Abgrenzung der Arbeiterklasse, die in der wirtschaftlichen Entwicklung und dem befürworteten System gründet, wird hier theoretisch wieder aufgehoben durch eine potentielle gesellschaftliche Gleichstellung, die im liberalen Denken durchaus nicht Fiktion bleiben musste. In diesem Sinne etwa drückte sich Friedrich Albert Lange aus, wenn er in seiner Schrift „Die Arbeiterfrage” davon sprach, dass die Arbeiterschaft im Ganzen immer auf einer unteren Stufe bleiben werde, und dann hinzufügte: „... aber er (der Arbeiter, d.V.) kann doch seine Lage allmählich verbessern, und jeder kann sich, wenn er Muth und Verstand hat, zum Unternehmer emporschwingen."2 So erschien die Arbeiterklasse zwar im liberalen Wirtschaftssystem notwendig, brauchte aber in ihrer Zusammensetzung durchaus nicht festgelegt zu sein, sondern konnte sich völlig beweglich zeigen. Dass die Selektionstheorie Darwins in liberalen Kreisen auf fruchtbaren Boden fiel, wird aus obigen Darlegungen verständlich. ${ }^{3}$

Der Versuch, die Grenzen zwischen Unternehmer und Arbeiter auf dem Wege der Selbsthilfe zu überwinden, setzte zum anderen auch eine Anerkennung des manchesterlichen Wirtschaftssystems und seiner Spielregeln seitens der Arbeiterschaft voraus. Der Staat durfte zur Beseitigung von Missständen nicht aufgerufen werden, wie ohnehin Wirtschaft und Staat als zwei scharf zu unterscheidende und zu trennende Institute gelten mussten. Die Politik der Fortschrittspartei gerade hinsichtlich des allgemeinen Wahlrechts zeigte so auch, dass sie sich vornehmlich um den Einbau der Arbeiterschaft in das Wirtschaftsgefüge bemühte. Das Verhältnis der Arbeiter zum Staat sollte ein „bürgerliches” werden. Und die Situation war den Liberalen insofern günstig, als die Arbeiterschaft kein eigenes politisches Bewusstsein aus der Zeit von 1848 in die Jahre der Neuen Ära und des Gründeroptimismus hinübergerettet hatte. 4 Diesem Mangel der Arbeiter begegnete auf der Seite der Liberalen die fast ausschliess-

1 Vgl. dazu noch Lassalle, V S. 98 ff. Ziegler an Lassalle. 28.1 2.63.

${ }^{2}$ Friedrich Albert Lange, Die Arbeiterfrage in ihrer Bedeutung für Gegenwart und Zukunft (1865) S. 137 .

${ }^{3}$ Auch den Kantianer Lange wird man von solchen Gedanken nicht freisprechen können.

Vgl. dazu Reisig, a.a.O. S. 87 und 94. 
liche Hinwendung zu wirtschaftlichen Problemen, die die politischen Idealisten $z u$ wirtschaftspolitischen Realisten wandelte, wobei darauf hingewiesen werden muss, dass ja weniger die Intelligenz und das handwerkliche Kleinbürgertum der $48 \mathrm{er}$ Jahre als vielmehr die neue Unternehmerschicht das Denken des Bürgertums bestimmte. Der Begriff des Liberalen reduzierte sich so auf die Wirtschaft. Sie war das zentrale Gebiet politischer und gesellschaftlicher Gestaltung. So versuchte man nicht nur, die Arbeiterbildungsvereine unpolitisch zu halten, sondern zog sich imgrunde selbst aus der politischen Sphäre zurück, um jedoch bald aus dieser Klausur mit Gedanken politisch wieder hervorzutreten, denen unverkennbar eine Verquickung von wirtschaftlichem Erfolg und politischer Forderung zugrundelag. Schon der Mangel einer festen Organisation der Fortschrittspartei darf darauf schliessen lassen, dass es keinesfalls um eine Politisierung des Bürgertums um jeden Preis ging. Die Opposition gegen den Partikularismus, der Kampf um ein nationales Deutschland, konnte lange nicht alle Bereiche der Politik erfassen.

Es war dies auch der Punkt, an dem Hans Baumgarten mit der "Selbstkritik des Liberalismus" ansetzte, und man wird wohl annehmen dürfen, dass diese Kritik sowohl unter dem Eindruck der zielbewussten Politik Bismarcks als auch der Organisation der lassalleanischen Bewegung unter Schweitzer geboren wurde. So versuchte Baumgatten, das Bürgertum an seine politischen Pflichten zu mahnen, indem er auf die Unzulänglichkeit des extremen „Idealismus” und „Materialismus” hinwies. Aber zugleich begrenzte er politische Fähigkeit und politische Urteilskraft wieder auf jene Schichten, die sich durch Besitz und Bildung als ,fähige Glieder der Gesellschaft" bewiesen. Und das darf als eine Formulierung gelten, die dem Erwerbsbürgertum jener Jahre geläufig war. Verquickung von Besitz und Bildung: aus dieser Grundhaltung heraus werden auch die Äusserungen von Unruhs und Rochaus über mangelnde Reife und Bildungsunfähigkeit der besitzlosen Arbeiterschaft zu verstehen sein. Mehr unter dem Aspekt der Verbindung von Besitzlosigkeit und Bildung nun wird auch der allgemeine Wunsch, mittels der Arbeitervereine die Bildung der Arbeiterschaft zu heben, gesehen werden müssen. So war es im Jahresbericht des Erfurter Vereins formuliert worden, so wurde es auch auf den Vereinstagen immer wieder hervorgehoben. Das Motto von der Selbsthilfe implizierte Anerkennung des liberalen Wirtschaftsweges und somit auch die Kenntnis von der Notwendigkeit der Arbeiterklasse im anerkannten System. Bildung und Wissen schienen geeignet, den Mangel materieller Güter ideell auszugleichen, vielleicht sogar zu beseitigen. Dass es dabei nicht 
um politische Bildung ging, erhellt aus zahlreichen Äusserungen und Erklärungen im Bezug auf das Programm der Vereine, wiewohl man in linken Kreisen durchaus die Hoffnung hegte, auf dem Wege über die "gründliche Bildung" zu einer günstigen politischen Situation für die Arbeiterschaft zu gelangen, die eine Trennung von der Bourgeoisie einbegriff. So konnte Rudolf Schramm im Oktober I 863 an Moses Hess schreiben: „Die Besserung der politischen Organisation der Gesellschaft scheint mit unvermögend, das Schicksal der Volksmassen umzugestalten, wenn nicht gleichzeitig mit ihr die Besserung der unter dem Drucke dieses Schicksals gegenwärtig verkommenen Einzelmenschen vorwärtsschreitet. Wenn die Arbeiter bleiben, was sie sind und nur die Herren der politischen Situation werden, so ist damit ihnen selbst noch dem Ganzen geholfen. Sie werden eben verzehren und aufbrauchen, was sie an erworbenem Kapital der alten gesellschaftlichen Ordnung vorfinden... Will man die Arbeitermassen daher von der Bourgeoisie ablösen, so darf man sie nicht principaliter auf den Staat, sondern nur auf die eigene Intelligenz, Energie und Sittlichkeit verweisen. Diese zum Teil schlummernden Potenzen zu sollizitieren und in Tätigkeit zu setzen, ist eine Aufgabe, welche nicht verschoben werden darf, bis die Arbeiter die politischen Herren geworden sein werden." 1 Es mag dabei unbeachtet bleiben, ob hier Schramm in den Arbeiterbildungsvereinen eine geeignete Institution sah, seine Konzeption zu verwirklichen, deutlich wird jedenfalls nach einem Studium des Weges der sozialdemokratischen Partei, dass jener aus den Arbeiterbildungsvereinen hervorgehende Eisenacher Zweig der gesamten Bewegung das politische und schliesslich auch im Hinblick auf die Rezeption des Marxismus ideologische Gesicht gab.

Sich des Politischen nun völlig zu enthalten, erschien schlechterdings unmöglich, und dass es hier und da zu ebenso zaghaften wie unklaren Ansätzen in der Diskussion politischer Aufgaben kam, weiss sich Bebel aus seiner Tätigkeit im Leipziger Verein „Vorwärts” zu erinnern. ${ }^{2}$ Jedoch wusste man den Begriff des Politischen recht restriktiv auszulegen. Auf dem ersten Vereinstag von $186_{3}$ liess man darüber keinen Zweifel aufkommen, als man sagte, dass man sich auch politischen Dingen zuwenden wolle (Allgemeine Volkswirt-

1 Lassalle, a.a.O., V S. 248 f. 29.10.1863.

2 So schrieb er: „Diese Erörterungen waren noch sehr unklar. Man diskutierte über die Invalidenversicherung der Arbeiter, über die Veranstaltung einer Weltausstellung in Deutschland, über den Eintritt in den Nationalverein... weiter forderte man das allgemeine Stimmrecht für die Landtagswahlen und ein deutsches Parlament, das sich der Arbeiterfrage anzunehmen habe. Ferner wurde die Einberufung eines allgemeinen deutschen Arbeiterkongresses diskutiert, auf dem die aufgetauchten Forderungen debattiert werden sollten." I S. 54 . 
schaftslehre, Verfassung des eigenen und fremder Staaten), aber Beschäftigung mit Politik nicht politische Agitation bedeuten könne. ${ }^{1}$ Ziel und Zweck der Arbeiterbildungsvereine erfuhren hier eine deutliche Abgrenzung gegen die in diesen Monaten einsetzende Agitation Lassalles. Die Kenntnis des Bestehenden wurde gefordert. Sie implizierte zugleich eine Bestätigung. Auf solchem Hintergrund lässt sich Lassalle verstehen, wenn er sagte, dass er allgemeines Wahlrecht meine, wenn er von Revolution rede.

Im naturwissenschaftlich-technischen Bereich geboten die Arbeitervereine Erweiterung der einschlägigen Kenntnisse. Das entsprach durchaus den Forderungen der zügigen industriellen Entwicklung, die in Deutschland noch soviel gegenüber den Nachbarstaaten aufzuholen hatte. So machte die Bedienung der Maschinen grössere Kenntnisse auf technischem Gebiet erforderlich, als sie bisher - wenn überhaupt - vorhanden war. Es wurde gerade in jenen Jahren besonders deutlich, wie schlecht es mit der Volkserziehung in elementaren Wissensbereichen bestellt war. Stimmen tauchten auf, die sogar zwingende Verpflichtung zur Fortbildungsschule forderten. Dabei ist jedoch nicht gesagt, dass die Arbeitervereine das naturwissenschaftlich-technische Programm auf die Forderungen der praktischen Fabriktätigkeit einengten und so mehr den Charakter von Berufsund Fachschulen annahmen. Vielmehr ging der Rahmen der Unterweisung weit über die engen Fachgrenzen hinaus, und es mag hier als Beispiel nicht unerwähnt bleiben, dass in jenen Jahren Brehms "Tierleben" aufgelegt wurde ( $1864-69) .^{2}$ So wie man sich der Wissenschaft in der materiellen Produktion bediente und sie ihr nutzbar machte, ging es darum - und darauf hat Hildegard Reisig schon mit Recht hingewiesen -, den Arbeiter zum völligen Verständnis der wissenschaftlichen Welt und ihrer Grundlagen zu führen, um die entfremdende manuelle Arbeitsleistung zu durchgeistigen und ihr den Charakter schöpferischer Tätigkeit zu geben. ${ }^{3}$ Inwieweit sich diese Einheit von geistig-schöpferischer und manuell-mechanischer Arbeit als Fiktion erwiesen hat, kann hier nicht unserer Beurteilung unterliegen.

Es ist deutlich geworden, dass jener quantitativen Aneignung naturwissenschaftlich-technischer Fakten die qualitative Umwandlung zum

1 Die Verhandlungen des 1 . Vereinstags der deutschen Arbeitervereine zu Frankfurt am Main. 7. u. 8. Juni 1863. S. I 2. „Politischen Unterricht nach solchem Vorbild zu erteilen, heisst aber noch lange nicht, Politik und politische Agitation treiben."

2 Das Werk wurde auch die "Tierbibel" des deutschen Volkes genannt. Es ist dabei noch interessant zu wissen, dass Prof. Rossmässler, einer der warmen Befürworter der Arbeitervereine, mit Brehm 1866/67 „Die Tiere des Waldes” herausgegeben hat.

${ }^{3}$ Vgl. Reisig, a.a.O. S. 97 . 
Selbstverständnis des Arbeiters folgen sollte. Das bedeutete schon einen ersten Schritt aus dem Bereiche der rein materiellen Produktion in das Gebiet der Persönlichkeitsbildung und somit einen Schritt vom Betriebstechnischen zum Gesellschaftspolitischen. So leuchtet es auch ein, dass das Bildungsziel letztlich staatspolitischen Charakter tragen sollte. Staatspolitische Erziehung im liberalen Sinne bedeutete Sorge um eine bejahende Haltung des Arbeiters zur politisch-sozialen Realität, die als eine Stufe auf dem Wege des Fortschritts erkannt wurde. Mit der Definition der gegebenen Verhältnisse als notwendiger Stufe des Fortschritts war imgrunde die Rechtmässigkeit politischer Tätigkeit des Arbeiters theoretisch widerlegt, soweit sie sich ausserhalb der Vereine und somit des gesamten Erziehungssystems ein Betätigungsfeld suchen wollte. Dem Versuch, das Denken der Arbeiterschaft in bürgerliche Bahnen zu lenken, entsprach zugleich der Wunsch, dem Arbeiter anhand eines Kataloges normativer Sittlichkeit den Leitfaden zu geben. Aus der Niederschrift über die Tagung des $\mathrm{r}$. Vereinstages lässt sich entnehmen, wie sehr die alten bürgerlichen Tugenden diesen Katalog ausfüllten. So hiess es da: (nachdem man über die allgemeine Unwissenheit der Arbeiter gesprochen hatte, d.V.) „... Dazu kommt noch der Mangel an grösserer Strebsamkeit und Sparsamkeit, wie ferner der Mangel an Sinn für die höheren Werte des Lebens, für Sitteneinfachheit und Sittenreinheit, für Benehmen und Charakter." 1 Und zu diesen Punkten stiess dann die nationale Formel, die in der Revolution von I 848 vom Bürgertum schon einmal ausgesprochen worden war und nunmehr auch die Arbeiterschaft in nationaler Funktion sehen wollte. „Aus dem Ganzen heraus aber tritt als mächtige Blüte," so schrieb man auf dem Vereinstag nieder, „die Liebe zur Freiheit und zum Vaterlande, die beide ihren Ausdruck finden in einem freien allgemeinen deutschen Staatsbürgertum, in einem einigen und freien deutschen Vaterland"'.2

Idealistische Bildungsgedanken in Verbindung mit staatspolitischen Erziehungswünschen, wirtschaftspolitische Überlegungen und parteipolitische Bemühungen formten so die Grundlage der Arbeiterbildungsvereine, die bei aller anerkennenswerten Leistung die politische Aktion der Arbeiterschaft nicht einzuschränken vermochten. Dass aus dem Bildungsverein "zur materiellen und gesellschaftlichen Förderung des Einzelnen" bald die Partei hervorging, die etwa I Jahrzehnt später begann, den Marxismus zu rezipieren, und sich als „Exponent einer Gesellschaftsschicht fühlte", 3 war von Beginn an

1 1. Vereinstag $186_{3}$ S. 5 .

2 Ebd. S. 7.

3 Vgl, zu diesen Erörterungen Reisig, a.a.O. S. ros. 
eine mögliche Konsequenz der Entwicklung gewesen. In dem Masse wie lerneifriges Studium die so oft beklagte Unwissenheit zurückdrängte, nahm auch die Bewusstseinsformung $\mathrm{zu}$, und es ist deutlich, dass den Arbeitern angesichts der interessenpolitischen Formierung weiter Fortschrittskreise die eigene Klassensituation bewusster wurde, was alte Bildungsidealisten wie Rossmässler ihre Aufgabe nicht erfüllen liess.

Zugleich mit jener inneren Entwicklung zur politischen Partei sahen sich die Vereine Angriffen von aussen, insbesondere Lassalles, ausgesetzt, der zu seinen Lebzeiten zwar die bürgerliche Ruhe der Vereine nicht direkt gefährden konnte, wohl aber Zweifel an der Ehrlichkeit ihrer Bildungsprogramme zu streuen vermochte. Die politische Agitation des Bürgers Lassalle stellte sich gegen die politische Passivität der ebenso von Bürgern organisierten Bildungsvereine!

\section{IV}

Gustav Mayer schreibt einmal zur politischen Aktion Lassalles: „Es hat dem Verständnis von Lassalles historischer Erscheinung auf der einen Seite gedient, es auf der anderen Seite aber auch aufgehalten, dass man sie ständig mit dem Phänomen Marx verglichen hat. Denn damit presste man sie in ein Schema, in das sie nicht hineinpasste, und unterwarf sie Massstäben, die auf sie nicht zutrafen." ${ }^{1}$ In der Tat führt ein solcher Vergleich zu einer Kritik beider Systeme, die natürlich möglich, dem Verständnis des politischen Wirkens Lassalles aber insofern hinderlich ist, als den politischen Gegebenheiten und der politischen Aktion kein Raum gewährt wird. Das würde ein Verbleiben am Rande des Historischen bedeuten.

Es gilt zunächst festzuhalten, dass der $48 \mathrm{er}$ Liberale Lassalle sich in den 6oer Jahren mitten in den innenpolitischen Gegensatz zwischen Liberalen und Konservativen gestellt fühlte. Für die Liberalen lag der mögliche Weg zur Lösung der sozialen Frage in der Förderung von Arbeiterbildungsvereinen, auf deren unpolitischen Charakter man bedacht war. Der individualistische Selbsthilfe-Gedanke sollte Vereinsprogramm und Vereinspolitik bestimmen. Will man einmal von Schulze-Delitzsch's Genossenschaftsgedanken absehen, so erschöpfte sich das Bemühen der Fortschrittler um eine konstruktive Sozialpolitik in dem Ruf nach solchen Arbeiterbildungsvereinen. Demgegenüber vermochten konservative Kreise mit wesentlich konkreteren Plänen aufzuwarten. Die soziale Frage erschien ihnen ungleich dringlicher. Man wird hier auf Hermann Wagener hinweisen

${ }^{1}$ Gustav Mayer, Zum Verständnis der politischen Aktion Lassalles. In: International Review for Social History, IlI (1938) S. 89. 
müssen, dem Wolfgang Saile jüngst eine aufschlussreiche Studie - Verhältnis zu Bismarck und sozialreformerische Tätigkeit - gewidmet hat.

Wagener arbeitete in der unmittelbaren Umgebung Bismarcks. Seinem Drängen auf durchgreifende gesetzgeberische Behandlung der Frage lag ein ebenso grundsätzliches wie nüchternes politisches Kalkül zugrunde. Mehrere Denkschriften Wageners für Bismarck lassen deutlich werden, dass sich ihm als Grundproblem die allmähliche Abschwächung der zunehmenden revolutionären Tendenz der Arbeiterschaft stellte. Um die von ihm doch bejahte Ordnung der Gesellschaft zu bewahren, galt es einzugreifen, ehe das nun noch rein im sozialen Bereich verhaftete Problem zu einem politischen wurde, die politische Forderung sich zur sozialen gesellte.

Was Wagener als brennende Zeitfragen deutete, das war neben dem Arbeiter- vor allem das Handwerkerproblem. ${ }^{1}$ Es blieb ihm nicht verborgen, dass sich die Arbeiterschaft in steigendem Masse aus ehemaligen Handwerkern rekrutierte. Um der korporativen Gesellschaftsordnung einen festen Boden zu geben, verlangte er vorab gesetzgeberische Hilfe zur Stütze der Handwerkerschaft. So zielte er vor allem auf die verschärfte Durchführung von Schutzbestimmungen $\mathrm{ab}$, die noch vom Februar 1849 datierten und als wesentlichsten Punkt die Abgrenzung der Gewerbe $(\$ 28)$ enthielten. Eine Massnahme dieser Art musste dann die Reorganisation der Gewerberäte zur Folge haben. Die Gewerberäte ihrerseits konnten dann als Grundlage für den weiteren Ausbau der gleichsam korporativen Selbstverwaltung (Errichtung von Gewerbegerichten) des Handwerkerstandes dienen.

Es ist deutlich, dass Wagener auf diesem Wege der möglichen Gefährdung und dem fortschreitenden Zerfall in der kapitalistischen Konkurrenzwirtschaft entgegenwirken wollte. Einer solchen Einengung des manchesterlichen Prinzips - imgrunde doch eine Abgrenzung nach beiden Seiten: zu den Unternehmern und zum Proletariat liess Wagener seine Vorschläge für die Arbeiterschaft hören. Die Punkte, welche hierbei namentlich in den Vordergrund traten, waren

„a. Die Feststellung eines Minimums von Tagelohn mit völliger Freigebung der Stück- und Akkordarbeit als Ausdruck der Wahrheit, dass auch in der Industrie die menschenwürdige Existenz das Eine und Wesentlichste ist.

b. Die gesetzliche Feststellung der Zusammengehörigkeit des Ar-

1 Siehe zu dem folgenden den Quellenanhang bei Wolfgang Saile, Hermann Wagener und sein Verhältnis zu Bismarck, (1958) S. I33 ff. Anlage I-III. 
beiters und der Fabrik und zwar so, dass der Arbeiter nicht mit der einzelnen Fabrik, sondern mit dem betreffenden Fabrikzweige in Verbindung gesetzt wird.

c. Die Anerkennung des Satzes, dass andauernde Mitarbeit auch Miteigentum verschaffen muss."1

Hier war weder die Rede von Selbsthilfe-Organisationen noch von Produktivgenossenschaften mit Staatshilfe. Wagener ging einen völlig eigenen Weg, der über die Anschauungen seiner konservativen Gesinnungsfreunde hinausführte. Ausdrücklich setzte er sich auch von Schulze-Delitzsch und Lassalle ab, den er in einer weiteren Denkschrift als einen gefährlichen Egoisten und verstandesmässigen Sozialisten charakterisierte, „der, wie die 'Reaktion' ihn, so seinerseits die Reaktion für seine Zwecke und als Übergangsstadium auszunützen versucht". 2

$\mathrm{Zu}$ dem korporativen Sozialstaat Wageners führte in der Tat kein Weg der Lassalleschen Vorschläge, zu welchem grundsätzlichen Punkte kommt, dass dem im Staate wirkenden und am Staate orientierten Wagener die heftige Agitation des Arbeiterführers zuwider sein musste. So sehr sich nun hier auch konservatives Ordnungsprinzip und sozialistischer Drang nach Neugestaltung gegenüberstanden, wie sehr auch Misstrauen und Abneigung herrschten, in einem Punkte vermochten sich Lassalle und Wagener zu begegnen: ihnen war der Widerstand gegen das Bürgertum, wie es sich in der Fortschrittspartei repräsentierte, gemeinsam. Wagener stand nicht an, von den „Oligarchen des Geldkapitals" zu sprechen, die das monarchische Prinzip bedrohten. Lassalle seinerseits genügte ein Rückblick auf die Erfahrungen der Revolutionsjahre I848/49, um fest die Meinung vertreten zu können, dass das Bürgertum zur festen tatkräftigen politischen Aktion nicht imstande sei. Dass in den sechziger Jahren „eine Partei wie die Fortschrittspartei noch möglich war, bewies ihm die Richtigkeit seiner Annahme."3 In diesem Sinne äusserte er sich in einem Brief an Wilhelm Rüstow, und es ist interessant zu wissen, dass sich drei Jahre später in liberalen Kreisen Stimmen erhoben, die selbstkritisch Lassalle beipflichteten. ${ }^{4}$

Dem politischen Agitator Lassalle, der, wie er einmal gegenüber Sophie von Hatzfeldt äusserte, "Wille als Weisheit" besass, ihm, dem die politische Aktion alles bedeutete, musste ein solcher Charakter von vornherein zuwiderlaufen. Ausgehend von dem oben bezeugten

1 Aus Anlage II, S. 137 .

2 Anlage III, I S. $142 \mathrm{f}$.

Mayer, Politische Aktion Lassalles S. 92.

4 Darüber, dass der Idealismus die politischen Fähigkeiten verdorben habe, siehe Baumgarten, a.a.O. S. 483 . 
Unpolitischen im Bürgertum, ist es aufschlussreich zu erfahren, dass sowohl Lassalle wie Wagener diese Anlage in ihre politischen Betrachtungen einbezogen. Dabei machten beide den Unterschied zwischen „Bürgertum” und „Bourgeoisie." So schrieb Wagener in seiner Denkschrift vom I. März I 864 an Bismarck: „Überwinden aber kann man die Opposition der Bourgeoisie nur dadurch, dass man einmal die berechtigten materiellen Forderungen des - von ihr unterschiedenen - Bürgertums befriedigt und dies eventuell hierdurch gewinnt. ${ }^{1}$ Zugleich sprach sich Lassalle in seiner Schrift „Die Wissenschaft und die Arbeiter" noch deutlicher aus. Ihm wurde der Grossbürger dann zum Bourgeois, wenn er „Besitz als rechtliche Bedingung für die Theilnahme an der Bestimmung des Staatswillens" machte. ${ }^{2}$

Während Wagener in seiner Wendung gegen die Bourgeoisie für die „Emanzipation von dem nachgerade unerträglichen Druck des finanziellen Feudalismus" eintrat, sich aber im übrigen doch mit seinen Vorschlägen im Rahmen des korporativen Sozialstaatsgedankens bewegte, stellte sich Lassalle mit aller Deutlichkeit auf den Boden des allgemeinen Wahlrechts, welches durchzusetzen eine festgefügte Arbeiterorganisation ermöglichen musste. Die politische Lösung der sozialen Frage stellte sich für Lassalle vorerst als das wesentliche Problem, ihm gab er in seiner Agitation den Vorrang. So wird man auch von dem Wunsch Lassalles nach Verwirklichung der Demokratie mit und durch die Arbeiterschaft ausgehen müssen, sollen Lassalle und Wagener in ihrem Verhältnis zueinander bewertet werden. In der neueren Literatur zögert Ernst Engelberg nicht, von einer ,intellektuellen Gevatterschaft" und von einer „politischen Konspiration" beider zu sprechen, ${ }^{3}$ indem er auf Gemeinsamkeiten in der Beurteilung des Bürgertums hinweist. Will man zunächst einmal von der "politischen Konspiration” absehen - das Verhältnis zu Bismarck ist ein für die Beurteilung dieses Punktes entscheidender Faktor - so scheint uns der andere, etwas grobe Begriff der ,intellektuellen Gevatterschaft" insofern nicht stichhaltig, als lediglich die Parallelität einiger Gedanken, nicht aber das Ziel Lassalles berücksichtigt wird. Und hier können doch tiefgreifende Unterschiede aufgezeigt werden.

Die wenigen Jahre des politischen Lebens Lassalles lassen den Arbeiteragitator dem Historiker problematisch genug erscheinen. Der Historiker wird hingelenkt auf den politischen Kampf Lassalles um

1 Angeführt bei Ernst Engelberg, Zur Entstehung und historischen Stellung des Preussich-deutschen Bonapartismus. Beiträge zum neuen Geschichtsbild [Sonderdruck o.J.] S. 242.

2 Ferdinand Lassalle, Die Wissenschaft und die Arbeiter ( 1863 ).

${ }^{3}$ Vgl. Engelberg, a.a.O. S. 243. 
das allgemeine Wahlrecht und findet sich in der Analyse der Entwicklung Eigentümlichkeiten des politischen Weges gegenüber, die sich aus dem bizarren Charakter der Person und den Umständen gleichermassen erklären lassen. Vom allgemeinen Wahlrecht führt der Weg zu Lassalles Auffassung vom politischen Wert der Arbeiterschaft, wobei zugleich die Meinung politischer Freunde und Symphatisierender deutlich werden kann. Er führt darüber hinaus zu dem Verhältnis Lassalle-Bismarck, das Lassalle nicht ganz zu Unrecht den argen Zweifel sozialdemokratischer Politiker eingetragen hat und das bei aller Kühnheit des politischen Kurses Mass und Möglichkeiten des Politikers Lassalle erkennen lässt. Und im weiteren wird hier auch ideologische Kritik ansetzen, die die Frage nach der Stellung des allgemeinen Wahlrechts in der sozialistischen Theorie stellen muss. Das heisst gleichzeitig die Frage nach dem Verhältnis von Revolution und parlamentarischer Tätigkeit stellen.

(Schluss folgt). 